\title{
Seismic behavior of a low-rise horizontal cylindrical tank
}

\author{
Alessandra Fiore ${ }^{1,2} \cdot$ Carlo Rago $^{3} \cdot$ Ivo Vanzi $^{1,3,4} \cdot$ Rita Greco $^{5} \cdot$ Bruno Briseghella $^{4}$
}

Received: 10 January 2018 / Accepted: 2 May 2018 / Published online: 12 May 2018

(c) The Author(s) 2018

\begin{abstract}
Cylindrical storage tanks are widely used for various types of liquids, including hazardous contents, thus requiring suitable and careful design for seismic actions. The study herein presented deals with the dynamic analysis of a ground-based horizontal cylindrical tank containing butane and with its safety verification. The analyses are based on a detailed finite element (FE) model; a simplified one-degree-of-freedom idealization is also set up and used for verification of the FE results. Particular attention is paid to sloshing and asynchronous seismic input effects. Sloshing effects are investigated according to the current literature state of the art. An efficient methodology based on an "impulsive-convective" decomposition of the container-fluid motion is adopted for the calculation of the seismic force. The effects of asynchronous ground motion are studied by suitable pseudo-static analyses. Comparison between seismic action effects, obtained with and without consideration of sloshing and asynchronous seismic input, shows a rather important influence of these conditions on the final results.
\end{abstract}

Keywords Cylindrical tank $\cdot$ Sloshing $\cdot$ Asynchronous seismic action $\cdot$ FE modeling $\cdot$ Seismic behavior

\section{Introduction}

Seismic loading can induce large damages in industrial facilities and their complex components (Babič and Dolšek 2016; Demartino et al. 2017a, b; Nuti et al. 2009). The loss of the structural integrity of these structures can have severe consequences on the population, the environment and the economy (Krausmann et al. 2010; Fiorentino et al. 2015; Rodrigues et al. 2017). Looking at power/chemical/ petrochemical plants, storage tanks containers are widely employed. These hold liquids, compressed gases or mediums used for the short- or long-term storage of heat or cold.

Alessandra Fiore

alessandra.fiore@poliba.it

1 InGeo, University of Chieti-Pescara "G. d'Annunzio", Viale Pindaro 42, 65127 Pescara, Italy

2 DICAR, Politecnico di Bari, Via Orabona 4, 70125 Bari, Italy

3 Next Innovation in Engineering, Spin-Off University of Chieti-Pescara "G. d'Annunzio", Seconda traversa Strettola Sant'Anna alle Paludi n. 11, 80142 Naples, Italy

4 College of Civil Engineering, Fuzhou University, Fuzhou 350108, Fujian, China

5 DICATECh, Technical University of Bari, Via Orabona 4, 70125 Bari, Italy
Liquid storage tanks and piping systems are considered as critical components of those industrial facilities (Vathi et al. 2017; Bakalis et al. 2017).

The seismic response of tanks has been widely studied in the past starting from the pioneering studies of Housner (1957, 1963). In particular, Housner (1957) first presented the simplified formulae to compute the dynamic pressures developed on accelerated liquid containers and successively (Housner 1963) studied the dynamic behavior of groundsupported elevated water tanks considering equivalent spring-mass systems. Current practice for the seismic design of storage tanks is mainly based on Appendix E of API 650 (2007) standard and on Eurocode 8 (1998). Generally speaking, there are many different types of equipment used for the storage of liquids and gases. The characteristics of the different tanks adopted mainly depend on: (a) the quantity of fluid being stored, (b) the nature of the fluid, (c) the physical state of the fluid and (d) the temperature and pressure. In industrial plants, gases are usually stored under high-pressure, often in liquid form since the volume is largely reduced. Within this framework, ground-based horizontal cylindrical tanks resting upon two supports are used mainly for storage of various liquids. The capacity of such tanks considerably exceeds those of horizontal tanks designed for land transportation. Under the conditions of normal exploitation, such tanks are loaded mainly with internal pressure being the sum of hydrostatic 
pressure and the uniform pressure caused by vapor of the medium contained therein (Magnucki et al. 2004).

For a cylindrical pressure vessel, there are two possible failure modes. One is the maximum stresses reaching the yield condition and then yielding zone spread leading to final plastic collapse and the other is elastic or elastic-plastic buckling leading to collapse. For the first problem it is mainly a stress analysis while for the second problem it is a stability analysis. In particular this instability appears usually in two forms: the elephant foot buckling and the diamond buckling (Niwa and Clough 1982; Haroun and Bhatia 1994; Hamdan 2000). The first form, which is an outward bulge located just above the tank base, results from the combined action of vertical compressive stresses, exceeding the critical stress, and hoop tension close to the yield limit. The second form is an elastic instability phenomenon due to the presence of high axial compressive stresses.

For sake of brevity, this study exclusively deals with stress analysis, while stability analysis is not documented.

More in detail, the present paper analyses the seismic performances of a ground-based horizontal cylindrical tank containing "butane", focusing on the two topics of sloshing (1) and asynchronous seismic input (2).

As to the first aspect (1), it is worth noting that the seismic analysis of cylindrical storage tanks requires accounting for the fluid-structure interaction. This phenomenon, referred to as "liquid sloshing," is generated by the presence of a free surface allowing for fluid motions and is generally caused by external tank excitation, significantly affecting in many cases the dynamic response (Hamdan 2000; Patkas and Karamanos 2007).

As to the second aspect (2), asynchronous motion denotes the differences in amplitude, phase and frequency content among ground motions recorded over extended areas (Nuti and Vanzi 2005; Lavorato et al. 2017). This spatio-temporal variation of ground motion is mainly attributed to (Zerva 2009; Koufoudi et al. 2018): (a) difference in arrival times of seismic waves at different locations; (b) loss of coherence of seismic waves (i.e. gradual reduction of its statistical dependence on distance and frequency), due to multiple reflection and refraction as they propagate through the highly inhomogeneous soil medium; (c) ground motion attenuation; (d) impact of local site effects. According to the current Italian and European technical codes (M.I.T 2008, Eurocode 8), if foundations are not properly interconnected with sufficiently rigid elements, asynchronous motion has to be accounted for.

The analysis herein presented comprises a sophisticated numerical FE modeling as well as a simplified model for the estimation of the dynamic properties of the tank structure. The paper is organized as follows: First, the steel cylindrical pressure vessel containing butane adopted for the case study is presented ("Case-study" section). "Sloshing" and "Asynchronous seismic input and resulting structural demand" sections describe the mathematical model adopted for accounting for the sloshing and the asynchronous seismic input, while "Verification of finite element modeling" section focuses on the fundamental period of the structure. Results of the analyses are given in "Stress analysis" section; finally, conclusions are given in "Conclusions" section.

\section{Case-study}

The research focuses on an existing ground-based horizontal cylindrical steel vessel resting upon two r.c. supports through steel saddles and containing pressurized butane (density $\rho_{\mathrm{L}}=603 \mathrm{~kg} / \mathrm{m}^{3}$ ). The cylinder is $14.66 \mathrm{~m}$ long, with external diameter and thickness equal to $4 \mathrm{~m}$ and $12 \mathrm{~mm}$, respectively. The lateral sides of the cylinder are constituted by curvilinear surfaces; a reinforcing steel ring can be observed at the middle of the cylinder (Fig. 2).

The geometrical and mechanical characteristics of the cylindrical vessel are summarized in Tables 1 and 2 .

By observing Table 2, it can be noted that a rather low design strength (15 MPa) is attributed to r.c. supports. This choice was made for sake of safety since, in the absence of suitable test survey on the tank under examination, test results on adjacent vessels had highlighted low quality concrete.

Seismic input is given using the acceleration response spectrum defined according to the Italian code (M.I.T 2008). The following design conditions are adopted:

- nominal expected life of the structure: $V_{\mathrm{n}}=50$ years;

- utilization coefficient of the structure: 4th Class $\left(C_{\mathrm{u}}=2\right)$;

- reference period for the seismic action: $V_{\mathrm{R}}=100$ years;

- behavior factor: $q=1$.

Seismic zone is identified by the following characteristics: ground type: C; soil type $T_{1}(S=1.5)$.

Seismic hazard parameters of the site are given by:

Table 1 Geometrical properties of the cylindrical vessel

\begin{tabular}{ll}
\hline External diameter of the cylinder, m & 4 \\
Thickness of the cylinder walls, m & 0.012 \\
Height of the r.c. supports, m & 3.35 \\
Width of the steel saddles, $\mathrm{m}$ & 3.45 \\
Thickness of the steel saddles, $\mathrm{m}$ & 0.02 \\
\hline
\end{tabular}

Table 2 Mechanical properties of the cylindrical vessel

\begin{tabular}{lr}
\hline Design strength of cylinder steel $\left(f_{\mathrm{d}}\right)(\mathrm{MPa})$ & 360 \\
Design strength of supporting saddles steel $\left(f_{\mathrm{d}}\right)(\mathrm{MPa})$ & 230 \\
Design strength of r.c. supports $\left(f_{\mathrm{d}}\right)(\mathrm{MPa})$ & 15 \\
\hline
\end{tabular}


- design ground acceleration for the no-collapse requirement (ultimate limit state): $a_{\mathrm{g}}=0.053 \mathrm{~g}$;

- maximum amplification factor of the acceleration response spectrum: $F_{0}=2.571$;

- upper period of the constant acceleration branch of the response spectrum: $T_{\mathrm{C}}^{*}=0.512 \mathrm{~s}$.

The above values are representative of low seismicity areas in Italy (Vanzi et al. 2015; Fiorentino et al. 2018).

\section{Sloshing}

Seismic design provisions of liquid-storage tanks such as API 650 (2007) and Eurocode 8 (1998) are based on a mechanical spring-mass analogy initially developed by Graham and Rodriguez (1952), Jacobsen (1949) and Housner (1963) for rigid tanks and by Haroun and Housner (1982) for flexible tanks.

According to this analogy, a tank subjected to a seismic motion may be reduced to a simpler model with lumped masses and springs. More precisely a portion of the mass of the liquid content $\left(M_{\mathrm{I}}\right)$ is considered as rigidly connected to the tank walls while the remaining portion $\left(M_{\mathrm{C}}\right)$ is flexibly attached to the tank walls. The liquid (with mass $M_{\mathrm{I}}$ ) that synchronizes with the vibration of the tank is called impulsive while the sloshing component of the fluid (with mass $M_{\mathrm{C}}$ ), generating free surface waves and characterized by its own frequency of vibration, is referred to as convective component.

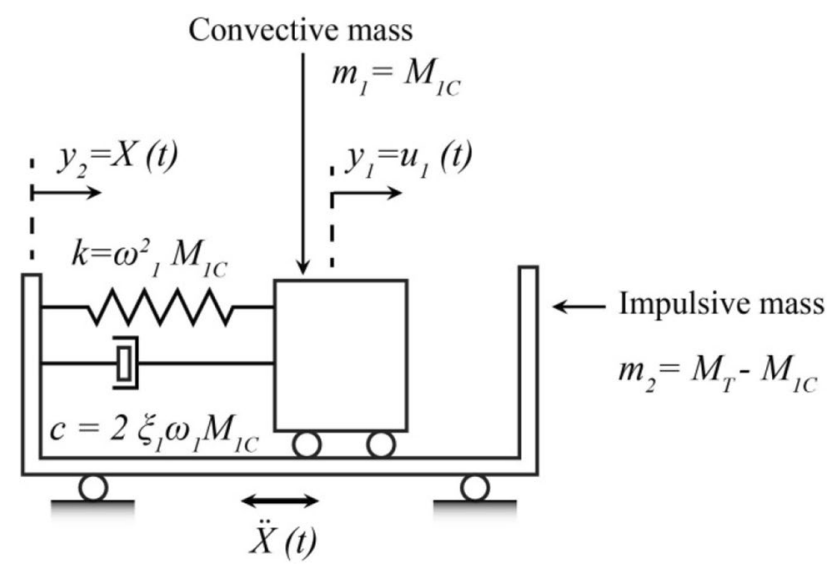

Fig. 1 Mechanical spring-mass analogy of a liquid-storage tank
In this study, the procedure from Karamanos (2004) is adopted to develop the mechanical spring-mass tank model. For broad tanks, the simplified model reported in Fig. 1 can be applied, where the tank-liquid system is represented by the first impulsive and first convective modes only. In fact, numerical calculations of hydrodynamic forces in horizontal cylinders and spheres showed that, in this case, considering only the first mode may provide a very accurate prediction of the convective and impulsive forces.

In Fig. 1, $y_{2}=X(t)$ represents the motion of the external source while $y_{1}=u_{1}(t)$ expresses the motion of the liquid mass associated to sloshing.

The total mass $M_{T}$ is split into two parts $m_{1}$ and $m_{2}$, corresponding to $y_{1}$ and $y_{2}$ and expressing the "convective" or "sloshing" motion $\left(M_{1 \mathrm{C}}\right)$ and "impulsive" motion $\left(M_{\mathrm{I}}\right)$, respectively.

As suggested by Eurocode 8 (1998), the seismic design force $F_{\mathrm{D}}$ can be calculated through the SRSS combination of the convective and impulsive maximum values $F_{\mathrm{C}, \max }$ and $F_{\text {I,max }}$ :

$F_{\mathrm{D}}=\sqrt{\left(F_{C, \text { max }}\right)^{2}+\left(F_{\mathrm{I}, \max }\right)^{2}}$.

The maximum convective $F_{\mathrm{C}, \max }$ and impulsive $F_{\mathrm{I}, \max }$ forces, neglecting the higher modes of vibration, are given by

$F_{\mathrm{C}, \max }=M_{1 \mathrm{C}} S_{\mathrm{A}}\left(T_{1 \mathrm{C}}\right) ; \quad F_{\mathrm{I}, \max }=M_{\mathrm{I}} S_{\mathrm{A}}\left(T_{\mathrm{I}}\right)$,

where $S_{\mathrm{A}}\left(T_{1 \mathrm{C}}\right)$ and $S_{\mathrm{A}}\left(T_{\mathrm{I}}\right)$ represent the spectral acceleration calculated in correspondence of the fundamental sloshing and impulsive periods, respectively.

The above quantities can be computed by utilizing the graphs and the tables reported in (Karamanos 2004), which refer to a cylindrical tank belonging to the same typology of the one herein analyzed. The procedure can be so summarized: (1) calculating the liquid mass $M_{\mathrm{L}}$ on the basis of the fluid level in the tank; (2) calculating the total moving mass $M_{\mathrm{T}}=M_{\mathrm{L}}+M_{\text {tank }}, M_{\text {tank }}$ being the mass of the empty tank; (3) deriving the convective mass $M_{1 \mathrm{C}}$ from Table 2 in (Karamanos 2004); (4) computing the total impulsive mass $M_{\mathrm{I}}=M_{\mathrm{T}}-M_{1 \mathrm{C}} ;(5)$ obtaining the fundamental sloshing period $T_{1 \mathrm{C}}$ and the fundamental impulsive period $T_{\mathrm{I}}$ from Tables 1, 2, 3,4 , and 5 in (Karamanos 2004). In the same Table 5 also the maximum convective force $F_{\mathrm{C}, \max }$, impulsive force $F_{\mathrm{I}, \max }$, and the total design force $F_{\mathrm{D}}$ for different liquid levels within the cylinder are reported. It can be noted that, since sloshing is a low-frequency motion, the corresponding spectral values are small and as a consequence, the impulsive component of the

Table 3 Dynamic properties of the cylindrical tank and seismic forces

\begin{tabular}{lllllllllll}
\hline$M_{L}$ [ton] & $M_{\text {tank }}$ [ton] & $M_{1 C}$ [ton] & $M_{I}$ [ton] & $T_{1 C}[\mathrm{~s}]$ & $T_{I}[\mathrm{~s}]$ & $S_{A}\left(T_{1 C}\right)\left[\mathrm{m} / \mathrm{s}^{2}\right]$ & $S_{A}\left(T_{I}\right)\left[\mathrm{m} / \mathrm{s}^{2}\right]$ & $F_{\mathrm{d}}[\mathrm{kN}]$ & $F_{C, \text { max }}[\mathrm{kN}]$ & $F_{I, \text { max }}[\mathrm{kN}]$ \\
\hline 108 & 29.14 & 25.134 & 112 & 1.73 & 0.3 & 0.61 & 1.65 & 185.8 & 15.332 & 185.24 \\
\hline
\end{tabular}


Table 4 Stiffness parameters of the SDOF model

\begin{tabular}{lllll}
\hline$M_{\text {eq }}($ ton $)$ & $k_{s t}(\mathrm{~N} / \mathrm{m})$ & $E\left[\mathrm{~N} / \mathrm{m}^{2}\right]$ & $J\left[\mathrm{~m}^{4}\right]$ & $H[\mathrm{~m}]$ \\
\hline 135.63 & $4.15 \times 10^{7}$ & $30 \times 10^{9}$ & 0.038 & 4.35 \\
\hline
\end{tabular}

Table 5 Pseudo-static analyses: maximum Von-Mises stresses

$\sigma_{\text {eff }}[\mathrm{MPa}]$

Soil differential displ. Soil differential displ. Soil differential displ. in $x$ dir. in $y$ dir. in $x$ and $y$ dir.

\begin{tabular}{lllllll}
\hline $\begin{array}{l}\text { Cylinder } \\
\text { walls }\end{array}$ & Saddles & & $\begin{array}{l}\text { Cylinder } \\
\text { walls }\end{array}$ & Saddles & $\begin{array}{l}\text { Cylinder } \\
\text { walls }\end{array}$ & Saddles \\
\hline 52.93 & 53.67 & 45.23 & 31.24 & 55.18 & 58.77 \\
\hline
\end{tabular}

response prevails. Thus the maximum seismic design force, i.e. the most unfavorable condition, is obtained in corresponding of the maximum possible liquid fill height in the cylinder, that is when the fluid mass tends to behave like an impulsive mass and sloshing effects become negligible.

\section{Application to the case study}

On the basis of the above considerations, the seismic analysis of the cylindrical tank object of study was carried out under the most unfavorable hypothesis of maximum seismic force, that is with the cylinder filled with butane up to the "block level" (i.e. the maximum allowable liquid level in the tank for safety reasons) equal to $80 \%$ in height. The $80 \%$ filling height corresponds to the $85 \%$ filling volume. Table 3 shows the deriving values of the involved parameters.

From Table 3, by comparing the values of $F_{\mathrm{D}}, F_{\mathrm{C} \text {,max }}$ and $F_{\text {I,max }}$, it can be deduced that the convective component of the fluid motion is negligible. So dynamic spectral analyses were carried out by modeling the liquid mass through its impulsive component only. In this way, an accuracy higher than $99 \%$ was obtained.

\section{Verification of finite element modeling}

The fundamental period of the cylindrical tank was determined by adopting two different approaches:

1. a detailed finite element (FE) model;

2. a simplified methodology based on a SDOF analogy.

The structure was assumed perfectly constrained at the basis.

As to the first approach (Resta et al. 2013), the tank was modeled by the FE structural analysis code Midas Gen 2017. Different typologies of FEs were used (Fig. 2): (1) plate elements to model the cylinder walls; (2) solid elements to model the steel saddles and the r.c. supports. A fundamental period equal to $0.2643 \mathrm{~s}$ in the $x$ (longitudinal) direction and 0.1376 in the $y$ (transversal) direction was so obtained (Fig. 3).

With regard to the second approach, the vessel was assimilated to a SDOF system with the mass given by the sum of four contributions: the cylinder steel mass; the saddles steel mass; part (1/3) of the mass of r.c. supports; the butane impulsive mass. The overall stiffness was schematized by a parallel-system of springs (Fig. 4). More precisely it was achieved by considering the stiffnesses $\left(k_{\mathrm{st}}\right)$ of the vertical r.c. supports arranged in parallel. By recalling that the stiffness, $k_{\text {eq }}$, of a parallel system is given by the sum of the different stiffness aliquots, it can be written as follows:
Fig. 2 FE model of the cylindrical tank

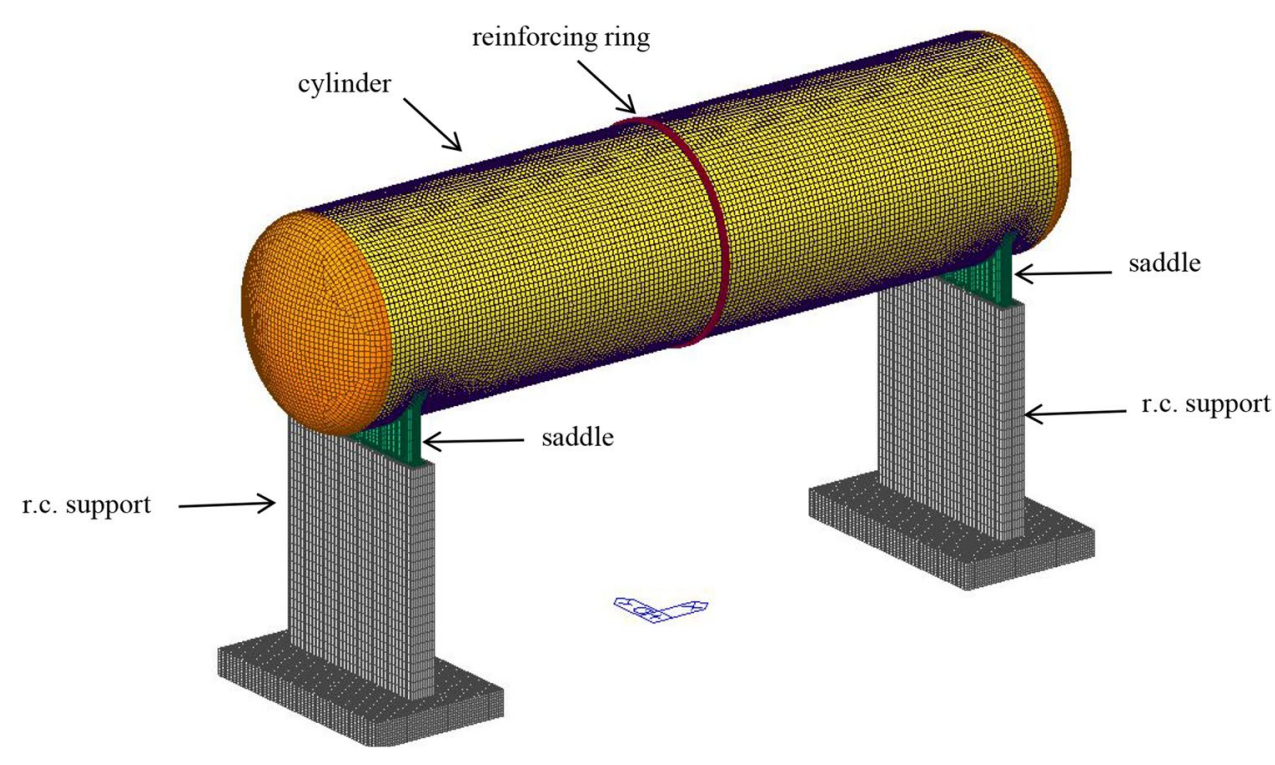



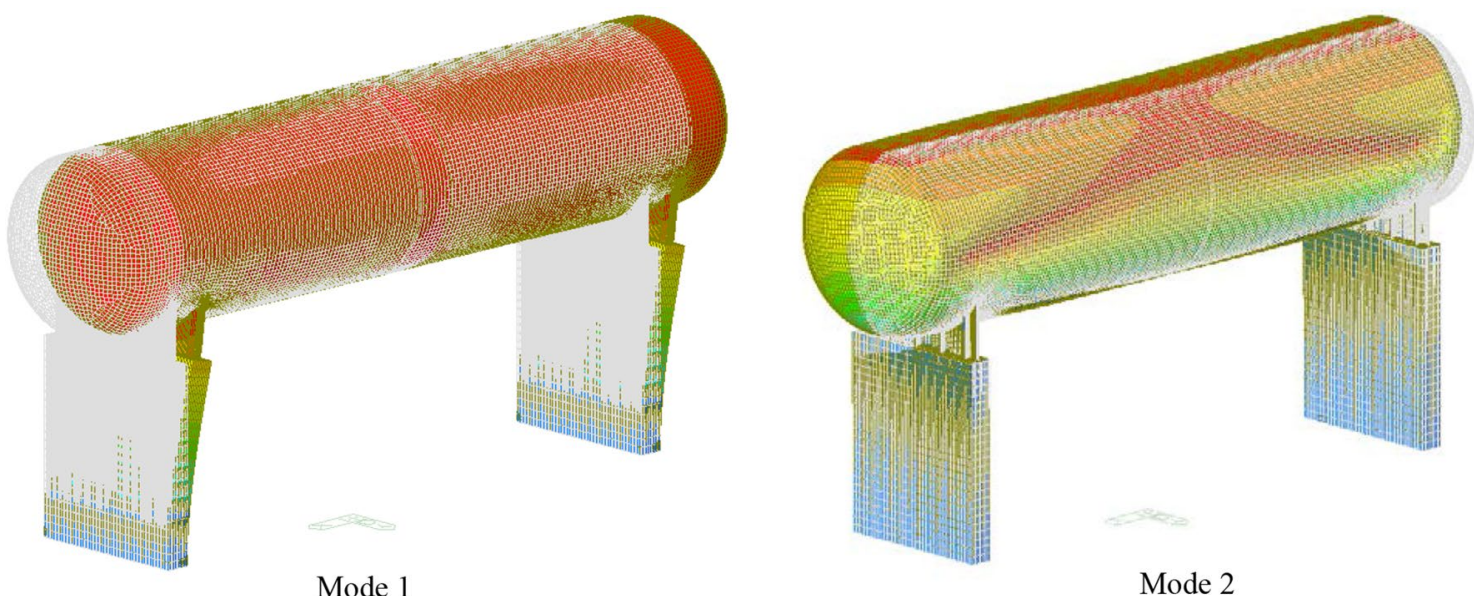

Mode 2

Fig. 3 First two modes of vibration of the cylindrical tank

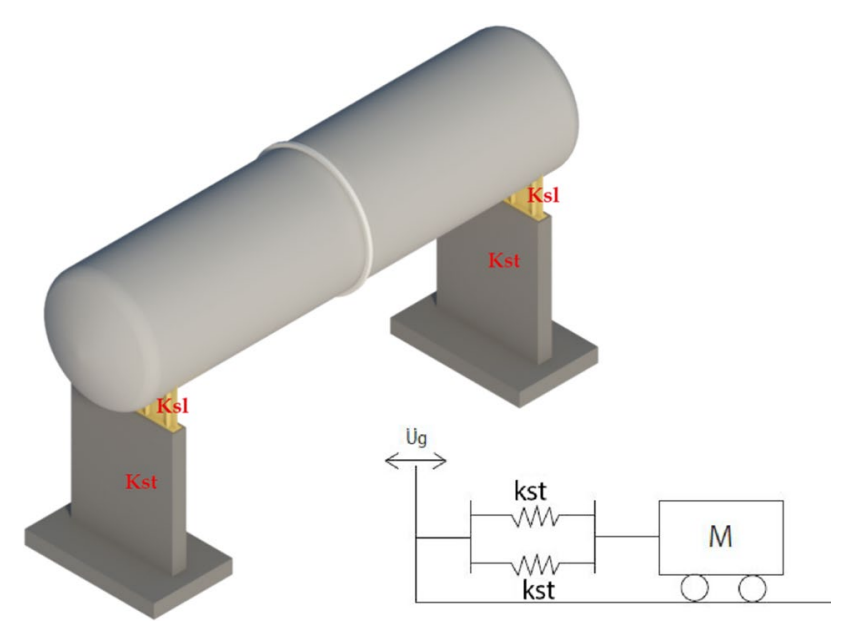

Fig. 4 SDOF analogy of the cylindrical tank

$T=2 \pi \sqrt{\frac{M_{\text {eq }}}{k_{\text {eq }}}} ; \quad k_{\text {eq }}=2 k_{\text {st }} ; k_{\mathrm{st}}=\alpha \frac{E J}{H^{3}}$.

Assuming $\alpha=3$, a fundamental period in the longitudinal direction equal to $0.254 \mathrm{~s}$ was computed, in perfect accordance with the value obtained through the FE model. The values of the various quantities involved in the analysis are summarized in Table 4.

\section{Asynchronous seismic input and resulting structural demand}

According to the current Italian code (M.I.T 2008, section 7.2.5.1) and to Eurocode 8, if foundations are not properly interconnected with sufficiently rigid elements, the effects of relative horizontal displacements at the basis of the superstructure should be analyzed in order to account for possible asynchronous actions.

The cylindrical vessel under examination belongs to this typology and thus the effects of non-synchronism were considered by introducing suitable soil differential displacements in the $x$ and $y$ directions at the foundation base. The differential displacements in the two directions were calculated according to section 3.2.5.2 of Italian code, excerpted from Nuti and Vanzi (2005), so obtaining in both directions $d_{i j}(x)=7 \mathrm{~mm}$.

Pseudo-static analyses were first carried out singularly in the $x$ and $y$ directions (Figs. 5,6), while in a second step soil distortions were introduced simultaneously in the two directions (Fig. 7). In all cases significant high stresses arose in the supporting saddles and in cylinder walls in correspondence of saddles, so highlighting that asynchronous actions can sensibly affect the stress level of the cylindrical tank and cannot be neglected. The maximum Von-Mises stresses obtained by the pseudo-static analyses are summarized in Table 5.

\section{Stress analysis}

The stress analysis was performed using the FE model of the cylindrical pressure tank depicted in "Verification of finite element modeling" section. The following load cases were considered:

- dead load $\left(G_{1}\right)$;

- internal pressure $\left(P_{\mathrm{i}}=6\right.$ bar $)$;

- hydrostatic pressure $\left(P_{\mathrm{H}}\right.$, due to butane);

- seismic spectral loads in all three directions. 


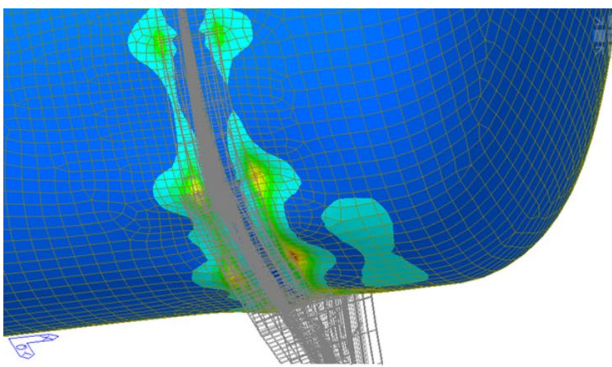

(a)

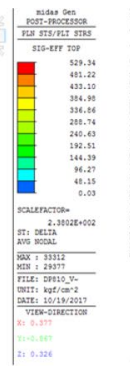

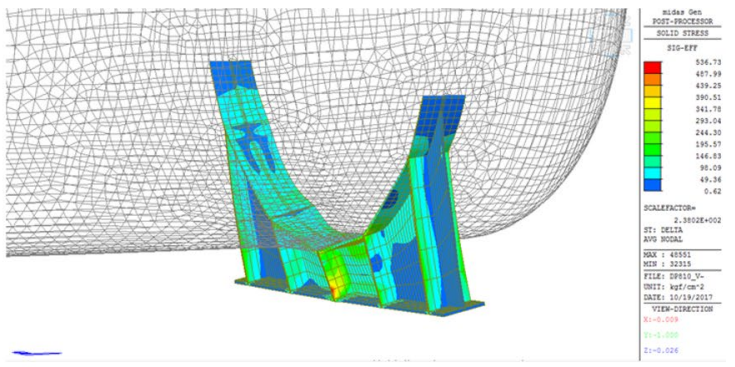

(b)

Fig. 5 Stress configuration due to soil differential displacements in the $x$ direction (Von-Mises stresses): a cylinder walls; b supporting saddle

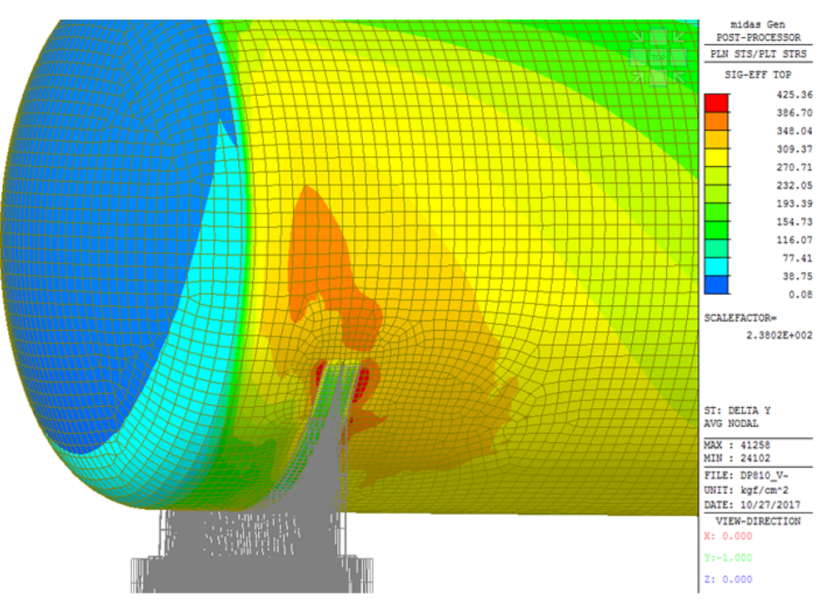

(a)

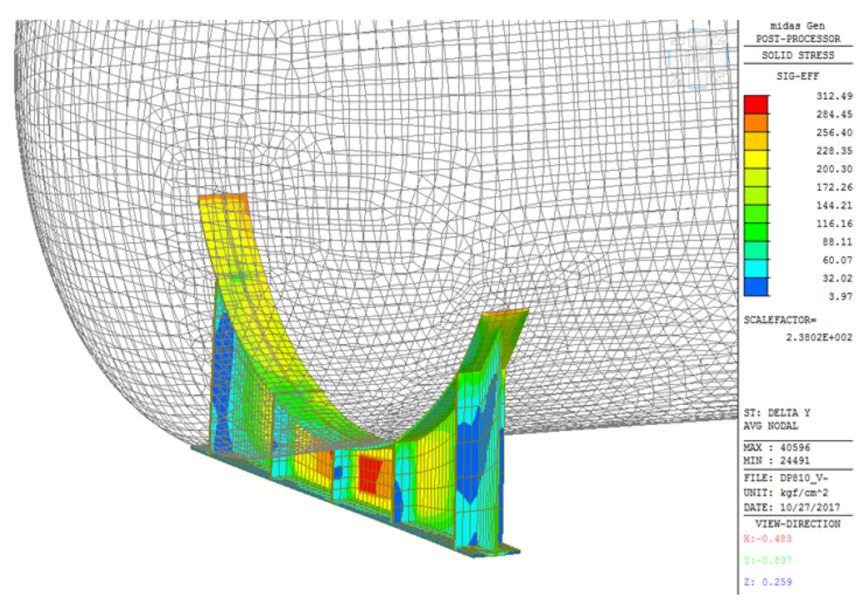

(b)

Fig. 6 Stress configuration due to soil differential displacements in the $y$ direction (Von-Mises stresses): a cylinder walls; b supporting saddle

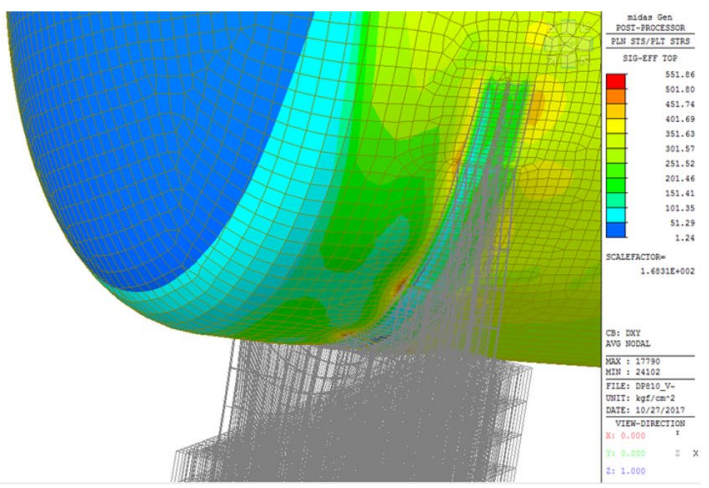

(a)

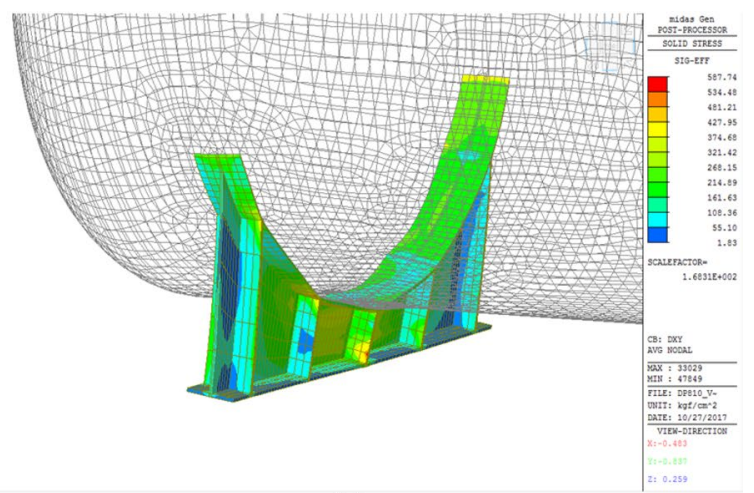

(b)

Fig. 7 Stress configuration due to soil differential displacements in both $x$ and $y$ direction (Von-Mises stresses): a cylinder walls; b supporting saddle 

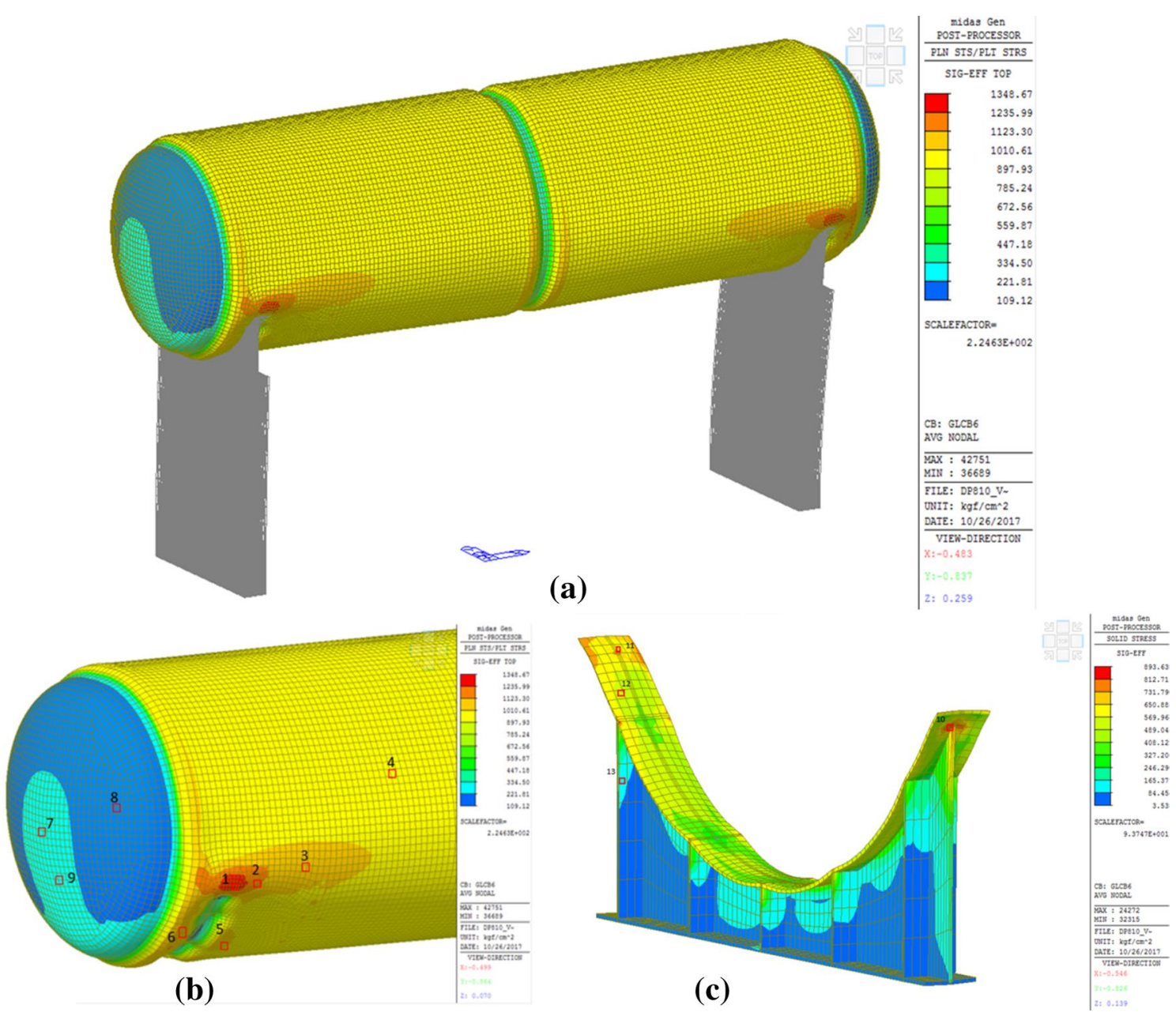

Fig. 8 Stress configuration in correspondence of the most unfavorable seismic load combination (Von-Mises stresses): a, b cylinder walls; $\mathbf{c}$ supporting saddle

Table 6 Stress verifications (Von-Mises stresses; maximum values compared with strength values)

\begin{tabular}{|c|c|c|c|c|}
\hline Element & $\begin{array}{l}\sigma_{\text {eff }} \\
\mathrm{MPa}\end{array}$ & $\begin{array}{l}f_{\mathrm{d}} \\
\mathrm{MPa}\end{array}$ & $\begin{array}{l}\text { Ratio } \\
\beta\end{array}$ & Verification \\
\hline Cylinder lateral walls & 134.3 & 360 & 2.68 & Satisfied \\
\hline Cylinder vertical walls & 22.8 & 230 & 10.09 & Satisfied \\
\hline Supporting saddles & 89.4 & 230 & 2.57 & Satisfied \\
\hline
\end{tabular}

Verifications were carried out in terms of Von-Mises stresses. The following load combination resulted to be the most unfavorable condition:

$1 \cdot G_{1}+1 \cdot P_{\mathrm{i}}+1 \cdot P_{\mathrm{H}}+0.3 \cdot \operatorname{Seism} X+1 \cdot \operatorname{Seism} Y+0.3 \cdot \operatorname{Seism} Z$.

Figure 8a-c shows the corresponding stress distributions; in particular Fig. 8a, b highlights stresses in the cylinder while Fig. $8 \mathrm{c}$ focuses on stresses in supporting saddles. Maximum stress values are reported in Table 6, while Table 7 focuses on stresses in some selected nodes, depicted in Fig. 8b, c. Maximum stresses arise at the interface between supporting saddles
Table 7 Stress verifications in selected FE nodes (in terms of Von-Mises stresses)

\begin{tabular}{llllllllllll}
\hline Nodes (from Fig. 8) & 1 & 2 & 3 & 4 & 7 & 8 & 9 & 10 & 11 & 12 & 13 \\
\hline$\sigma_{\text {eff }}(\mathrm{MPa})$ & 134.3 & 118.9 & 105.5 & 93.4 & 22.8 & 20.7 & 22.6 & 89.4 & 78.4 & 37.5 & 11.7 \\
$f_{\mathrm{d}}(\mathrm{MPa})$ & 360 & 360 & 360 & 360 & 230 & 230 & 230 & 230 & 230 & 230 & 230 \\
Ratio $\beta$ & 2.68 & 3.03 & 3.41 & 3.85 & 10.08 & 11.11 & 10.17 & 2.57 & 2.93 & 6.13 & 19.66 \\
\hline
\end{tabular}


and cylinder walls, that is in corresponding of the constraint sections, where, in absence of section increments, a stress increase is reasonable.

As it emerges from Tables 6 to 7, in all elements maximum stresses are lower than the corresponding design limit strengths, that is the analyzed cylindrical tank has a good level of safety against seismic action. Verifications on RC supports are not documented due to the absence of experimental tests on steel reinforcement details. However, it can be argued that they behave like perfectly constrained shelves, mainly stressed in the $x$ direction, and at most the low seismic forces acting on them can lead to the early formation of plastic hinges at the basis, with low ductility involvement (Fiore et al. 2016; Imperatore et al. 2012; Lavorato and Nuti 2010; Lavorato et al. 2015; Zhou et al. 2015).

Displacement diagrams for the same load combination are also given in Fig. 9. Maximum displacements result equal to: $1.99 \mathrm{~mm}$ in the $x$ direction; $3.4 \mathrm{~mm}$ in the $y$ direction; $1.33 \mathrm{~mm}$ in the $z$ direction.

Finally, in order to account for the effects of asynchronous actions, the response of the structure after the application of soil differential displacements (as described in "Asynchronous seismic input and resulting structural demand" section) was combined with the inertial response. The following seismic combination resulted to be the most unfavorable one:

$$
\begin{aligned}
& 1 \cdot G_{1}+1 \cdot P_{\mathrm{i}}+1 \cdot P_{\mathrm{H}}+0.3 \cdot \operatorname{Seism} X-1 \cdot \operatorname{Seism} Y \\
& \quad+0.3 \cdot \operatorname{Seism} Z+1 \cdot \text { Delta_} \_x+1 \cdot \text { Delta_} y .
\end{aligned}
$$

The resulting stress configurations and maximum stresses are reported in Fig. 10 and Table 8 respectively, showing an increment of response with respect to the previous case.

It is worth to note that, when liquid storage tanks are founded on piles, in some cases soil-structure interaction could be beneficial, leading to a higher value of natural period and to reduced seismic spectral forces (Fiore et al. 2018). This effect could so diminish the stress increments due to asynchronous seismic motion.

\section{Conclusions}

In this study, the seismic behavior of a cylindrical pressure vessel containing butane was analyzed, accounting for the influence of sloshing effects and of asynchronous actions. Both a detailed FE model of the horizontal cylindrical tank and a simplified SDOF model were implemented. It was

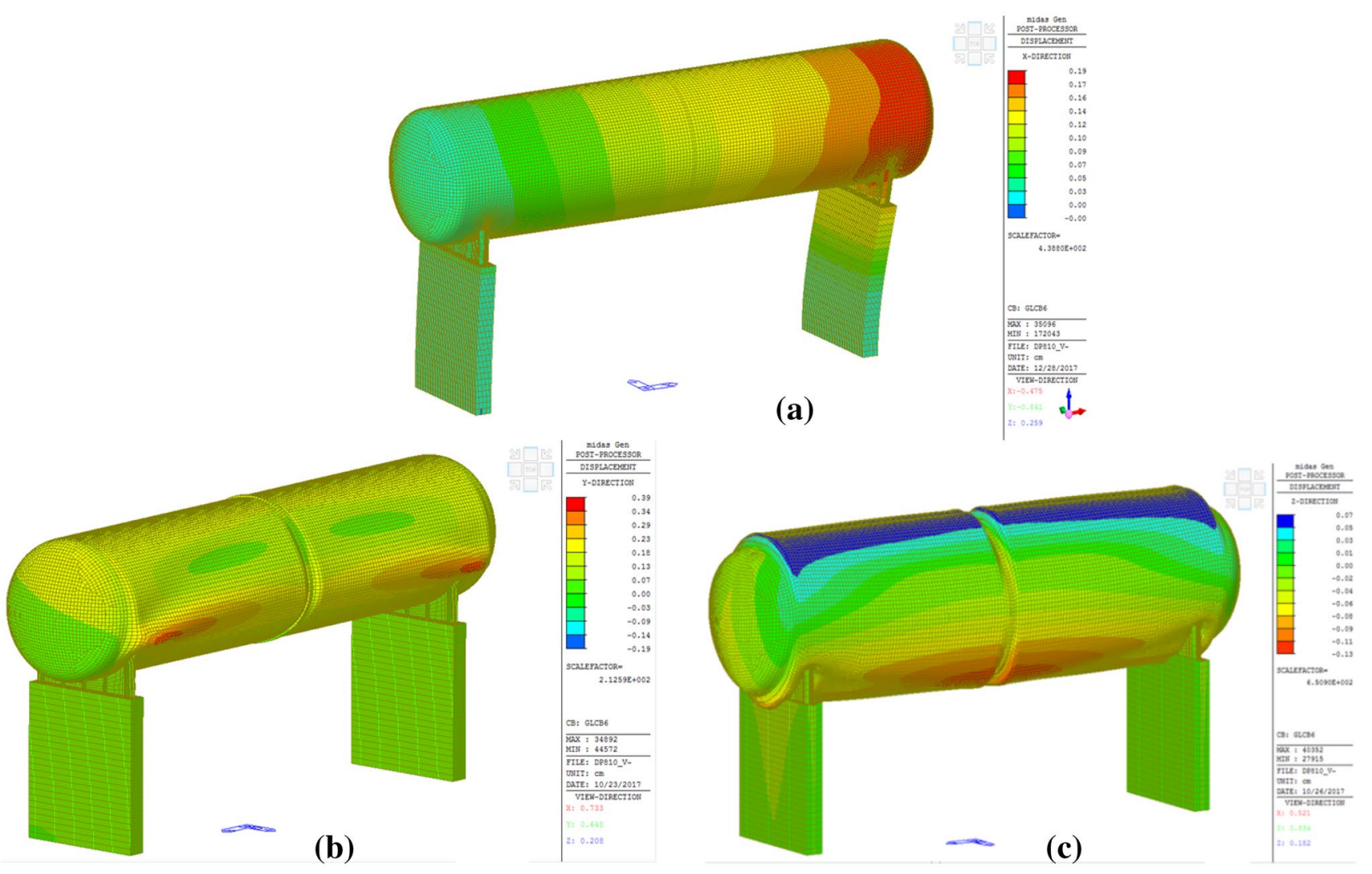

Fig. 9 Resultant displacements for the most unfavorable seismic load combination: a $x$ direction; b $y$ direction; $\mathbf{c} z$ direction 


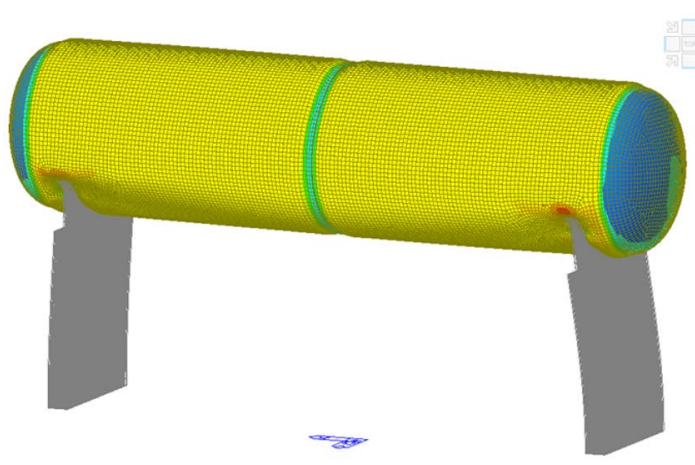

(a)

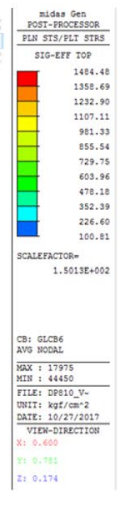

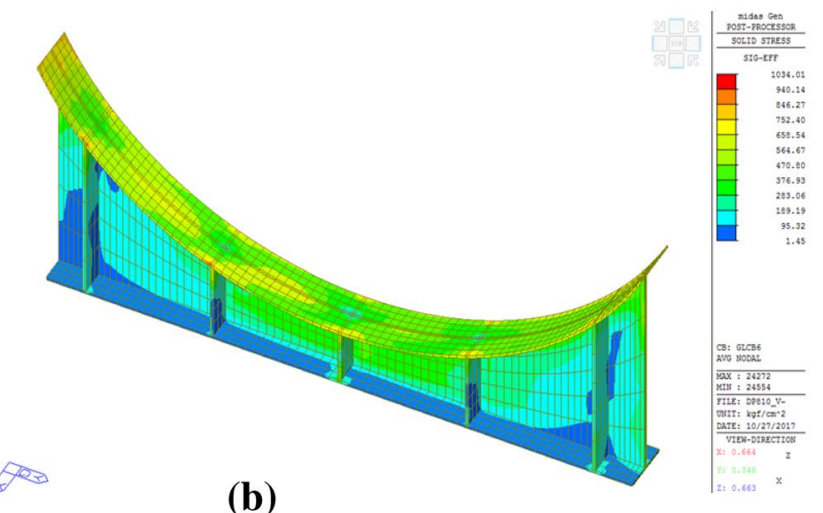

(b)

Fig. 10 Stress configuration including the effects of asynchronous ground motion (in terms of Von-Mises stresses): a cylinder walls; b supporting saddle

Table 8 Stress verifications including the effects of asynchronous ground motion (in terms of Von-Mises stresses)

\begin{tabular}{lllll}
\hline Element & $\begin{array}{l}\sigma_{\text {eff }} \\
\mathrm{MPa}\end{array}$ & $\begin{array}{l}f_{\mathrm{d}} \\
\mathrm{MPa}\end{array}$ & $\begin{array}{l}\text { Ratio } \\
\beta\end{array}$ & Verification \\
\hline Cylinder walls & 148.4 & 360 & 2.43 & Satisfied \\
Supporting saddles & 103.4 & 230 & 2.22 & Satisfied \\
\hline
\end{tabular}

shown which are the most unfavorable load conditions to be considered under sloshing and asynchronous input effects: (1) seismic action with liquid in the sphere up to the "block level"; (2) non-synchronism of ground motion in both $x$ and $y$ directions.

The high stress level associated with asynchronous seismic action was actually unexpected, given the low seismic design action. This was due to structural peculiarities, in which a very stiff and resistant system (the r.c. column) is connected to the tank via a welded steel saddle, which must accommodate, on a short length, the largest part of differential displacements. However, the possible steel yielding for higher seismic actions would not be a problematic issue for non-pressurized vessels; pressurized vessels, though, require larger reliability margins and simultaneous application of capacity design concepts, in order to satisfy structural safety when verified under large (larger than this paper design earthquake) seismic actions.

For the case of pressurized vessels, this is a reminder to check, even for low seismic action, for structural peculiarities (e.g. constraint behavior; coupling of very and moderately stiff/resistant components), which may become the weakest link for the whole structure, and, once identified, may quite often be structurally upgraded with modest efforts.

Open Access This article is distributed under the terms of the Creative Commons Attribution 4.0 International License (http://creat ivecommons.org/licenses/by/4.0/), which permits unrestricted use, distribution, and reproduction in any medium, provided you give appropriate credit to the original author(s) and the source, provide a link to the Creative Commons license, and indicate if changes were made.

\section{References}

API 650 (2007) Welded tanks for oil storage, 11th edn. American Petroleum Institute Standard, Washington, D.C.

Babič A, Dolšek M (2016) Seismic fragility functions of industrial precast building classes. Eng Struct 118:357-370

Bakalis K, Vamvatsikos D, Fragiadakis M (2017) Seismic risk assessment of liquid storage tanks via a nonlinear surrogate model. Earthq Eng Struct Dyn 46(15):2851-2868

Demartino C, Vanzi I, Monti G, Sulpizio C (2017a) Precast industrial buildings in Southern Europe: loss of support at frictional beamto-column connections under seismic actions. Bull Earthq Eng. https://doi.org/10.1007/s10518-017-0196-5

Demartino C, Monti G, Vanzi I (2017b) Seismic loss-of-support conditions of frictional beam-to-column connections. Str Eng Mech 61(4):527-538

Eurocode 8 (1998) Part 4, Silos, tanks and pipelines. ENV 1998-4, European Committee for Standardization, Brussels

Fiore A, Spagnoletti G, Greco R (2016) On the prediction of shear brittle collapse mechanisms due to the infill-frame interaction in RC buildings under pushover analysis. Eng Struct 121:147-159

Fiore A, Demartino C, Greco R, Rago C, Sulpizio C, Vanzi I (2018) Seismic performance of spherical liquid storage tanks: a case study. International Journal of Advanced Structural Engineering. https://doi.org/10.1007/s40091-018-0185-1

Fiorentino G, Furgani L, Magliano S, Nuti C (2015) Probabilistic evaluation of DAM base sliding. In: COMPDYN 2015-5th ECCOMAS thematic conference on computational methods in structural dynamics and earthquake engineering, pp 836-844

Fiorentino G, Forte A, Pagano E, Sabetta F, Baggio C, Lavorato D, Nuti C, Santini S (2018) Damage patterns in the town of Amatrice after August 24th 2016 Central Italy earthquakes. Bull Earthq Eng 16(3):1399-1423

Graham W, Rodriguez AM (1952) Characteristics of fuel motion which affect air plane dynamics. J Appl Mech 19(3):381-388

Hamdan FH (2000) Seismic behaviour of cylindrical steel liquid storage tanks. J Constr Steel Res 53(3):307-333 
Haroun MA, Bhatia H (1994) Analysis of tank damage during the 1994 Northridge Earthquake. In: Proceedings of the 14th US conference on lifetime earthquake engineering ASCE, New York, pp 763-770

Haroun MA, Housner GW (1982) Dynamic characteristics of liquid storage tanks. J Eng Mech Div ASCE 108-EM5:783-818

Housner GW (1957) Dynamic pressure on accelerated fluid containers. Bull Seismol Soc Am 47(1):15-35

Housner GW (1963) The dynamic behaviour of water tanks. Bull Seismol Soc Am 53:381-387

Imperatore S, Lavorato D, Nuti C, Santini S, Sguerri L (2012) Shear performance of existing reinforced concrete T-beams strengthened with FRP. In: Proceedings of the 6th international conference on FRP composites in civil engineering, CICE

Jacobsen LS (1949) Impulsive hydrodynamics of fluid inside a cylindrical tank and of a fluid surrounding a cylindrical pier. Bull Seism Soc Am 39:189-204

Karamanos SA (2004) Sloshing effects on the seismic design of horizontal-cylindrical and spherical vessels. In: Conference paper in American Society of mechanical engineers, pressure vessels and piping division (Publication) PVP, January 2004, https://doi. org/10.1115/pvp2004-2912

Koufoudi E, Cornou C, Grange S, Dufour F, Imtiaz A (2018) Quantification of the amplitude variability of the ground motion in Argostoli, Greece. Variability of linear and non-linear structural response of a single degree of freedom system. Bull Earthq Eng. https://doi.org/10.1007/s10518-018-0313-0

Krausmann E, Cruz AM, Affeltranger B (2010) The impact of the 12 May 2008 Wenchuan earthquake on industrial facilities. J Loss Prev Process Ind 23(2):242-248

Lavorato D, Nuti C (2010) Seismic response of repaired bridges by pseudodynamic tests. Bridge maintenance, safety, management and life-cycle optimization - proceedings of the 5th international conference on bridge maintenance, safety and management, pp 2375-382

Lavorato D, Nuti C, Santini S, Briseghella B, Xue J (2015) A repair and retrofitting intervention to improve plastic dissipation and shear strength of Chinese RC bridges. In: IABSE conference, Geneva 2015. Structural engineering, providing solutions to global challenges report, pp 1762-1767

Lavorato D, Vanzi I, Nuti C, Monti G (2017) Generation of non-synchronous earthquake signals. In: Gardoni P (ed) Risk and reliability analysis: theory and applications. Springer series in reliability engineering, Springer, Cham, pp 169-198
Magnucki K, Lewinski J, Stasiewicz P (2004) Optimal sizes of a ground-based horizontal cylindrical tank under strength and stability constraints. Int J Press Vessels Pip 81:913-917

MIT (2008) D.M. 14/01/2008. Norme Tecniche per le Costruzioni. Ministero Infrastrutture e Trasporti, Roma, Italy

Niwa A, Clough RW (1982) Buckling of cylindrical liquid-storage tanks under earthquake loading. Earthq Struct Dyn 10:107-122

Nuti C, Vanzi I (2005) Influence of earthquake spatial variability on differential soil displacements and SDF system response. Earthq Eng Struct Dyn 34:1353-1374

Nuti C, Rasulo A, Vanzi I (2009) Seismic assessment of utility systems: application to water, electric power and transportation networks. Saf Reliab Risk Anal Theory Methods Appl Proc Joint ESREL SRA-Europe Conf 3:2519-2529

Patkas LA, Karamanos SA (2007) Variational solutions for externally induced sloshing in horizontal-cylindrical and spherical vessels. J Eng Mech 133(6):641-655

Resta M, Fiore A, Monaco P (2013) Non-linear finite element analysis of masonry towers by adopting the damage plasticity constitutive model. Adv Struct Eng 16(5):791-803

Rodrigues D, Crowley H, Silva V (2017) Earthquake loss assessment of precast RC industrial structures in Tuscany (Italy). Bull Earthq Eng. https://doi.org/10.1007/s10518-017-0195-6

Vanzi I, Marano GC, Monti G, Nuti C (2015) A synthetic formulation for the Italian seismic hazard and code implications for the seismic risk. Soil Dyn Earthq Eng 77:111-122

Vathi M, Karamanos A, Kapogiannis IA, Spiliopoulos KV (2017) Performance criteria for liquid storage tanks and piping systems subjected to seismic loading. J Pressure Vessel Technol. https:// doi.org/10.1115/PVP2015-45700

Zerva A (2009) Spatial variation of seismic ground motions. Modeling and engineering applications. Advances in engineering series, CRC Press, Taylor \& Francis Group

Zhou Z, Lavorato D, Nuti C, Marano GC (2015) A model for carbon and stainless steel reinforcing bars including inelastic buckling for evaluation of capacity of existing structures. COMPDYN 20155th ECCOMAS thematic conference on computational methods in structural dynamics and earthquake engineering, pp 876-886

Publisher's Note Springer Nature remains neutral with regard to jurisdictional claims in published maps and institutional affiliations. 